\title{
Spontaneous separation of two-component Fermi gases in a double-well trap
}

\author{
JunJun Xu and QIANg Gu (a) \\ Department of Physics, University of Science and Technology Beijing, Beijing 100083, China
}

PACS 03.75.Ss - Degenerate Fermi gases

PACS 67.85.Lm - Degenerate Fermi gases

\begin{abstract}
The two-component Fermi gas in a double-well trap is studied using the density functional theory and the density profile of each component is calculated within the Thomas-Fermi approximation. We show that the two components are spatially separate in the two wells once the repulsive interaction exceeds the Stoner point, signaling the occurrence of the ferromagnetic transition. Therefore, the double-well trap helps to explore itinerant ferromagnetism in atomic Fermi gases, since the spontaneous separation can be examined by measuring component populations in one well.
\end{abstract}

Introduction. - Itinerant ferromagnetism has long been one of the central research topics in condensed matter physics [1. Recently, developments of laser trapping and cooling of atomic gases with multiple components have ' greatly renewed and broadened the interest in this area, and the new research interest is mainly expressed in two aspects.

First, the ${ }^{87} \mathrm{Rb}$ atoms provide an opportunity to study the itinerant-boson ferromagnetism [2, 3. It is the itinerant-electron ferromagnetism that has been intensively studied in the context of condensed matter physics. That is a typical Fermi system. The ${ }^{87} \mathrm{Rb}$ gas comes as the first example of the ferromagnetic Bose system. Motivated ' by the experimental achievement, the ground state properties [4,5] and thermodynamics [6,7] of the Bose ferromagnet have been studied theoretically. It was shown that the Bose gas is much easier to exhibit ferromagnetism than the Fermi gas: In the former case, the ferromagnetic (FM) transition temperature is never below the Bose-Einstein condensation temperature regardless of the magnitude of the ferromagnetic coupling [6], whereas in the latter case the ferromagnetism can not be present unless the ferromagnetic coupling exceeds the Stoner point [1].

Second, cold atomic Fermi gas can be used to simulate mechanism of the itinerant-fermion ferromagnetism, a long unsolved question in condensed matter physics. So far, the Stoner model gives us a qualitative description of itinerant ferromagnetism [1]. According to this model, the

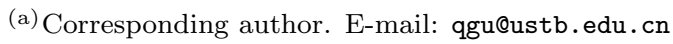

electron system can lower its total energy by spin polarization when the decrease of interacting energy is larger than the increase of kinetic energy due to Pauli principle. Since the interaction between atoms is tunable by Feshbach resonance 8 , people can examine whether such transition occurs when the repulsive interaction becomes stronger.

Recently an experimental group from MIT claimed their realization of ferromagnetic phase of Fermi gases in an equally populated mixture of ${ }^{6} \mathrm{Li}$ atoms in the lowest two hyperfine states [9]. They addressed the observation of non-monotonic behavior for increasing repulsive interactions, which implies the occurrence of itinerant FM transition through comparing with the Stoner's ferromagnetic mean-field theory. Previously, Duine and MacDonald had already investigated features of the FM transition based on second-order perturbation theory [10]. LeBlanc et al. studied observable experimental signatures of the FM transition theoretically within a local density approximation 11. More elaborate theoretical studies beyond the mean-field approximation showed that the FM transition could take place at a weaker interaction strength [12]. Moreover, various related problems, such as textured magnetization [13, pairing instability [14, spin fluctuations [15, and population imbalance [16], were investigated. On the other hand, the existence of a FM transition was questioned by Zhai, who suggested in a phenomenological way that a correlated state without ferromagnetism could also cause the similar features in the experiment [17.

Direct evidence of the FM transition in atomic gases 
might be the formation of magnetic domains, or the phase separation of different spin components. Amoruso et al. [18] and Salasnich et al. [19] calculated the density profile of two-component Fermi gases with conserved particle numbers and demonstrated the spatial symmetry breaking of the system. This means the formation of a sort of domain-structures. Furthermore, Sogo et al. indicated the similar phenomenon in an open system with variable particle numbers 20]. The MIT experiment failed in viewing the domain structure.

It is not an easy job to observe domain-structures in atomic gases directly. As Jo et al. claimed, the lifetime of domains might be too short and their size might be too small to be observed [21. Phase-contrast imaging has been successfully used to measure magnetization in situ in ${ }^{87} \mathrm{Rb}$ condensates [3, but in situ detection for atomic Fermi gases has not been reported as yet. Some recent theoretical proposals seek to verify the FM transition indirectly, e.g., by detecting topological signatures 22 and by calculating the spin drag relaxation rate 23 .

Here we propose an alternative method to detect the spatial symmetry breaking in the ferromagnetic Fermi gas. This proposal involves trapping the two-component atomic gas in the double-well (DW) potential. We show that the two components are spatially separated and each component prefers staying in one trap if the FM transition is induced by the strong repulsive interaction. This makes it convenient to determine the FM transition by measuring atoms in one well, in comparison to measuring magnetization in situ.

This paper is organized as follows. In section II, we calculate the ground state energy of two-component Fermi gases in a DW trap using the density functional theory. The kinetic term we are using is due to the so called Thomas-Fermi approximation as used by Sogo et al. 20. In section III, we show numerical results for the case with symmetric potential and equal particle numbers by analyzing the Euler equation. In Section IV, we consider the asymmetric case by changing the potential depth and the particle numbers, respectively. The last section shows our conclusions.

Basic model. -We now consider the ground state of two-component Fermi gases trapped in double wells at zero temperature. According to the density functional theory, the ground state energy of the system can be written as a functional of the densities:

$$
\begin{aligned}
E[\widetilde{n}(r)]= & \int d^{3} \widetilde{r}\left\{\sum_{\sigma=1,2}\left[\frac{\hbar^{2}}{2 m} \frac{3}{5}\left(6 \pi^{2}\right)^{\frac{2}{3}} \tilde{n}_{\sigma}^{\frac{5}{3}}+\widetilde{U}(\widetilde{r}) \widetilde{n}_{\sigma}\right]\right. \\
& \left.+g \widetilde{n}_{1} \widetilde{n}_{2}\right\}
\end{aligned}
$$

where the first term on the right hand corresponds to the kinetic energy of the system known as Thomas-Fermi approximation, and it has the similar form as the energy of uniform Fermi gases. The Thomas-Fermi approximation is considered a well approach near the center of the poten- tial trap for large total particle numbers [24]. The second term $\widetilde{U}(\widetilde{r}) \widetilde{n}_{\sigma}$ is the potential energy of the system within local density approximation, and

$$
\widetilde{U}(\widetilde{r})=\frac{1}{2} m \omega^{2}\left(\widetilde{x}^{2}+A e^{-B \widetilde{x}^{2}}+\widetilde{\rho}^{2}\right)
$$

describes the symmetric DW potential. Here $\widetilde{x}$ and $\widetilde{\rho}$ are the axial and radial coordinate. $A$ and $B$ are the constants related to the shape of the potential well, and $\sigma=1,2$ represents the two different component. $g \widetilde{n}_{1} \widetilde{n}_{2}$ is the interacting energy, where the strength of coupling is given by $g=4 \pi \hbar^{2} \widetilde{a} / m$ with $\widetilde{a}$ the s-wave scattering length. The interaction between the same component is absent as a result of the Pauli exclusion principle.

For simplicity, we transform the integral of equation (11) into cylindrical coordinate and then convert the parameters to dimensionless forms,

$$
\begin{array}{r}
\varepsilon=\alpha E, x=\beta \widetilde{x}, \rho=\beta \widetilde{\rho}, \\
n_{\sigma}=\zeta \widetilde{n}_{\sigma}, a=\gamma \widetilde{a},
\end{array}
$$

where

$$
\begin{aligned}
& \alpha=\frac{2^{17}}{3^{7} \pi^{7} \gamma^{8} m \omega^{2} \xi^{10}}, \\
& \beta=\frac{4}{3 \pi \gamma \xi^{2}}, \\
& \zeta=\frac{128}{9 \pi \gamma^{3}} .
\end{aligned}
$$

Here $\xi=\sqrt{\hbar / m \omega}$ is the oscillator length of the trap and $\gamma$ is a variable relevant to the density profile and the particle number, which is determined by

$$
N_{\sigma}=\int \rho d x d \rho n_{\sigma}=\frac{2^{12}}{3^{5} \pi^{5} \xi^{6}} \frac{1}{\gamma^{6}} \widetilde{N}_{\sigma},
$$

where $\widetilde{N}_{\sigma}$ is the number of each component and $N_{\sigma}$ is the reduced particle number.

Then equation (11) can be simplified as

$$
\varepsilon=\int \rho d x d \rho\left\{\sum_{\sigma=1,2}\left[\frac{3}{5} n_{\sigma}^{\frac{5}{3}}+U(x, \rho) n_{\sigma}\right]+a n_{1} n_{2}\right\},
$$

where $U(x, \rho)=x^{2}+c e^{-b x^{2}}+\rho^{2}$ is the reduced potential with $c, b$ are constants transformed from parameters $A$ and $B$, respectively,

$$
c=A \beta^{2}, \quad b=\frac{B}{\beta^{2}} .
$$

The ground state energy of the system can be obtained by minimize the energy functional. To ensure the conservation of particle number of each component, it's necessary to introduce two Lagrange multiplier $\mu_{1}$ and $\mu_{2}$, which just represent the reduced chemical potentials. The real chemical potential of the system $\widetilde{\mu}_{\sigma}$ reads

$$
\frac{\mu_{\sigma}}{\widetilde{\mu}_{\sigma}}=\frac{2^{5}}{9 \pi^{2} \gamma^{2} m \omega^{2} \xi^{4}} .
$$




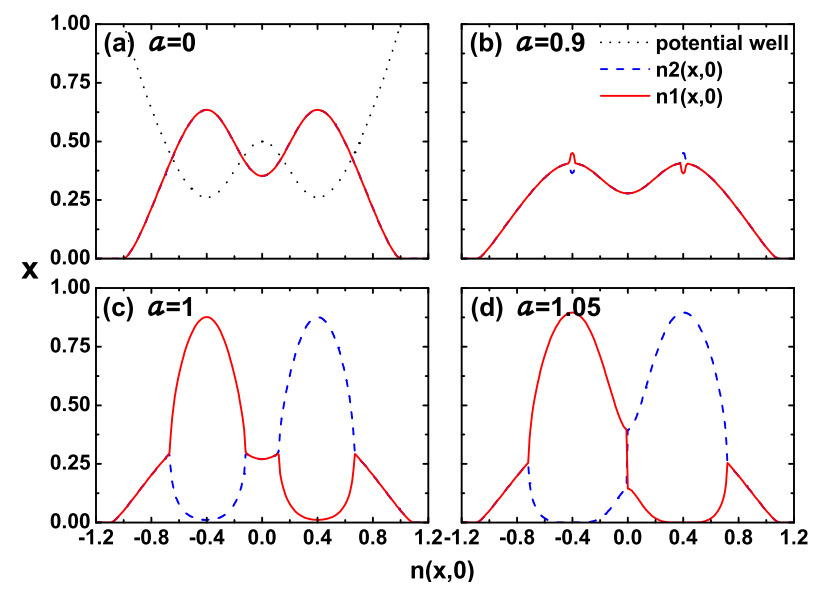

Fig. 1: Density profile along the axial coordinate $x$ with radial coordinate $\rho=0$. The reduced interaction strength $a$ from $(a)$ to $(d)$ is $0,0.9,1,1.05$. The dotted-black curve illustrates the potential well. The solid-red and dashed-blue curves represent different components.

The ground state energy has to fulfill the variational condition, $\delta\left(\varepsilon-\mu_{1} N_{1}-\mu_{2} N_{2}\right) / \delta n_{\sigma}=0$. So we obtain the following Euler equations,

$$
\begin{aligned}
n_{1}^{\frac{2}{3}}+a n_{2} & =M_{1}(x, \rho), \\
n_{2}^{\frac{2}{3}}+a n_{1} & =M_{2}(x, \rho),
\end{aligned}
$$

where $M_{\sigma=1,2}(x, \rho)=\mu_{\sigma}-\left(x^{2}+c e^{-b x^{2}}+\rho^{2}\right)$. By solving the Euler equations and keeping the particle number of each component conserved, we can get the density profile of each component in the ground state.

The symmetric case: with equal well depth and population. - In the case that the two-component fermions with equal population are confined in a symmetric DW trap, it is reasonable to assume the chemical potentials of the two components equal, $\mu_{1}=\mu_{2}=\mu$. For an ideal Fermi system the densities of the two components will be the same everywhere in the DW trap. In the presence of weak interactions, the density will not change much. This corresponds to the symmetric solution of Eqs. (11) and (12). In addition, Eqs. (11) and (12) have the asymmetric solution, which means that density profiles of the two components become different. If the asymmetric solution is stable, the symmetry of the ground state is spontaneously broken.

To minimize the ground state energy functional, one must make sure that $\partial^{2} \varepsilon / \partial n_{\sigma}^{2} \geq 0$. This demands that the following inequality holds everywhere in the DW trap,

$$
n_{1} n_{2} \leq\left(\frac{4}{9 a^{2}}\right)^{3} .
$$

By analyzing Eqs. (11) (13), the asymmetric solution is stable in case that the following condition is satisfied,

$$
M(x, \rho) \in\left[\frac{20}{27 a^{2}}, \frac{1}{a^{2}}\right] .
$$

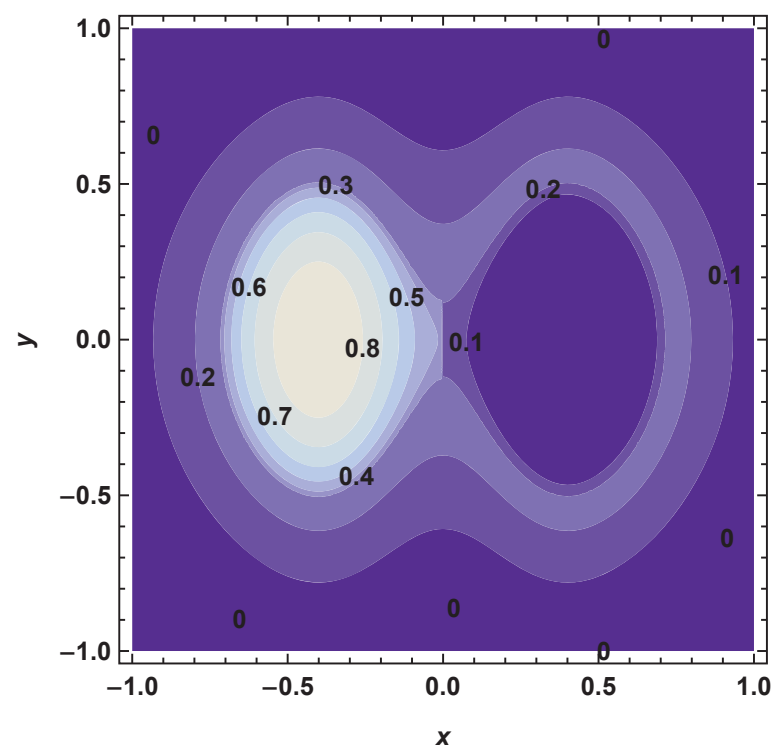

Fig. 2: Illustration of the density profile $n_{1}$ along the $x$ and $y$ coordinate with the interaction parameter $a=1.05$. Numbers in the figure show the values of the density.

There is only the symmetric solution when $0<M(x, \rho)<$ $\frac{20}{27 a^{2}}$, that is, $n_{1}=n_{2}$. Note that there is no stable solutions when $M(x, \rho)>\frac{1}{a^{2}}$ according to Eq. (13), which means that the two components are completely separated, so we have $n_{2}=0$ and the equation for $n_{1}$ becomes: $n_{1}=M(x, \rho)^{\frac{3}{2}}$.

In the whole article we consider a gas composed of ${ }^{6} \mathrm{Li}$ atoms with the trap frequency $\omega \approx(170 \times 2 \pi) \mathrm{Hz}$. First, the total number of two components is supposed to be $\widetilde{N}=$ $10^{6}\left(\widetilde{N}_{\sigma}=0.5 \times 10^{6}\right)$ and the DW potential is $U(x, \rho)=$ $x^{2}+0.5 e^{-10 x^{2}}+\rho^{2}$. Here the reduced particle number $N_{\sigma}$ is normalized to 0.1 for convenience and $\gamma \approx 2.57 \times 10^{6} \mathrm{~m}^{-1}$ correspondingly. The potential barrier is defined as $U_{0}=$ $\frac{1}{2} m \omega^{2} A$, then $U_{0} \approx 15.35 h \times \mathrm{KHz}$ according to Eqs. (9).

Figure 1 portrays density profiles of the two components. The spatial width of the confined Fermi gas and the distance between the two wells are about 2 and 0.8 . According to Eqs. (3), the corresponding physical values are about $119 \mu \mathrm{m}$ and $48 \mu \mathrm{m}$, respectively. It indicates that the symmetry-breaking solution becomes stable as long as the interaction strength is large enough. The two components tend to spatially separated and each component prefers to gather in one trap. That is the ferromagnetic state of two-component Fermi gas in double wells. This state begins to appear at about the interaction strength $a_{c} \approx 0.9$, corresponding to the critical scattering length $\tilde{a}_{c}=a_{c} / \gamma \approx 6600 a_{0}$, where $a_{0}=0.529 \AA$ is the Bohr radius. As the interaction strength increases, the two components become more separated. Note that the components are separated in the inside region of each trap, but are still equally mixed in the outside region. Figure 2 illustrates the density profile of one component along the $x$ and $y$ coordinate with $a=1.05$. 

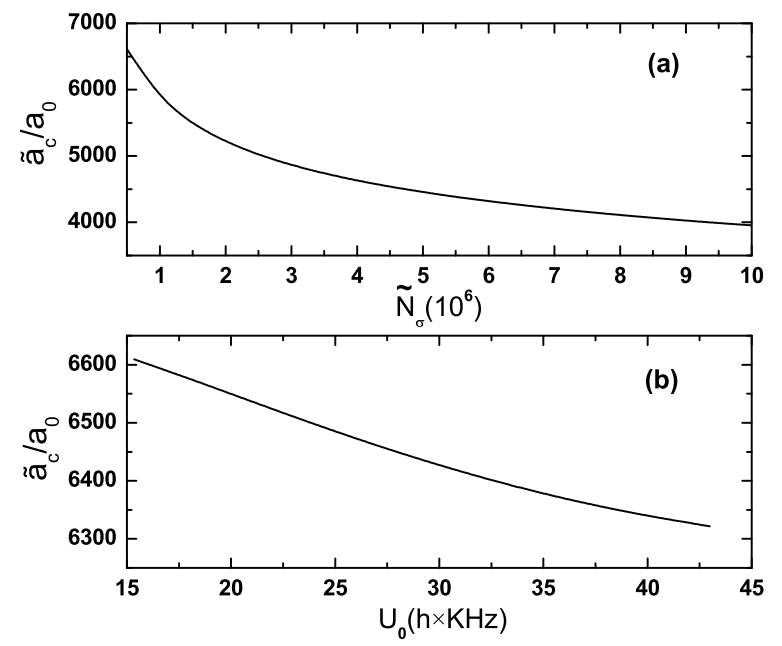

Fig. 3: The critical scattering length $\widetilde{a}_{c} / a_{0}$ as a function of the particle number $\tilde{N}_{\sigma}$ (a) and the barrier $U_{0}(\mathrm{~b})$. Here $a_{0}$ is the Bohr radius and $h$ is the Planck constant. $\widetilde{N}_{\sigma=1,2}=0.5 \tilde{N}$, where $\widetilde{N}$ is the total number.

LeBlanc et al. estimated that the critical value for $10^{6}$ ${ }^{6} \mathrm{Li}$ atoms confined in the single-well with the trap frequency $\omega \approx(170 \times 2 \pi) \mathrm{Hz}$ is about $6400 a_{0}$ [11. Therefore, trapping atoms in double wells does not cause much difficulty in generating the FM transition, in comparison to in the single-well trap. On the contrary, it helps the two components to be separate in two wells, and thus produces benefits for the detection.

Then we discuss the influence of the total particle number and the potential barrier to the Stoner point. Figure 3a shows that the critical scattering length $\widetilde{a}_{c}$ decreases apparently with the particle number increasing. Increasing the barrier $U_{0}$ but keeping the particle number $\widetilde{N}_{\sigma}=0.5 \times 10^{6}$ unchanged, the Stoner point is also lowered, but not that significantly, as shown in Fig. 3b. It is worth noting that there is no significant change in $k_{F}^{0} \widetilde{a}_{c}$ although $\widetilde{a}_{c}$ varies. $k_{F}^{0}=\left[6 \pi^{2} \widetilde{n}_{\sigma}(0)\right]^{\frac{1}{3}}$ is the Fermi wave vector of the interacting gas with $\widetilde{n}_{\sigma}(0)$ the density at the center of each well. Our calculation shows $k_{F}^{0} \widetilde{a}_{c} \approx 1.57$, which is consistent with the mean-field value in the uniform case, $k_{F}^{0} \widetilde{a}_{c}=\frac{\pi}{2}$.

The asymmetric case. - To proceed, we consider the asymmetric cases caused either by the population imbalance, or by the difference of well depth. The density profile can still be obtained by numerically solving Eqs. (11) and (12), with the particle number of each component being conserved. The problem of fermion mixtures in a single well with population imbalance has already been investigated [16.

Figure 4 shows the results for the unequal-population gas in the symmetric DW trap. The ratio of total numbers $N_{1}: N_{2}$ is $2: 1$. Similar to the equal population case, the two components distribute symmetrically in the two wells when the interaction strength is relatively weak. As

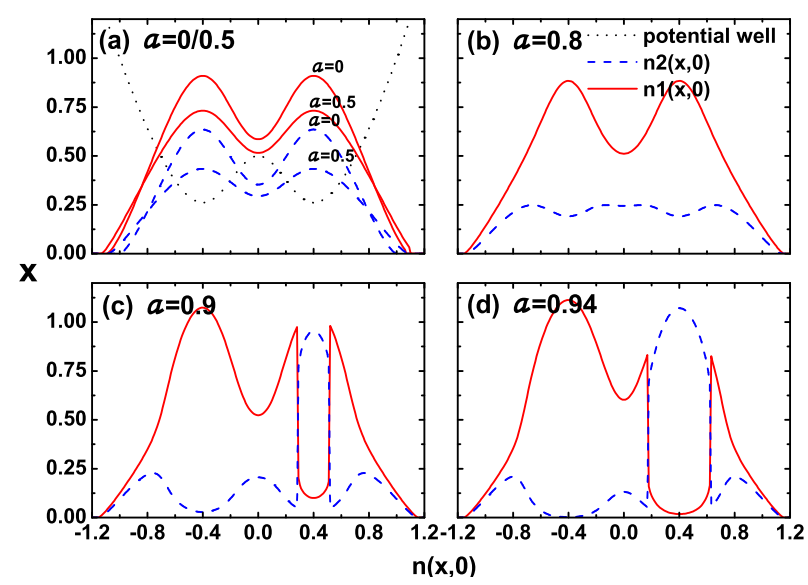

Fig. 4: Density profile along the axial coordinate $x$ with radial coordinate $\rho=0$ with unequal particle numbers $N_{1}=2 N_{2}=$ 0.2 . The reduced interaction strength $a$ from $(a)$ to $(d)$ is $0 / 0.5$, $0.8,0.9,0.94$. The dotted-black curve illustrates the potential well. The solid-red and dashed-blue curves represent different components.

the interaction grows stronger, this symmetric distribution becomes unstable. The two components tend to repel each other and each component begins to occupy the center of one potential well, signaling the occurrence of the FM transition.

Now look at a two-component Fermi gas with equal population $N_{1}=N_{2}=0.1$ in an asymmetric DW trap. The trapping potential is chosen as $U(x, \rho)=x^{2}+0.5 e^{-10 x^{2}}+$ $0.5 \sin (0.5 x)+\rho^{2}$. The third term in the right hand makes the trap asymmetric in the $x$-direction. As indicated in Fig. [5 the density of each component equals anywhere in the double trap in the weak interaction case, although they distribute asymmetric. As the interaction strength grows, the spatial separation of the two components occurs first in the deeper well, then in both wells. This phenomenon is easy to understand since the deeper well traps more atoms with larger density, so the interacting energy density is higher than that in the shallower well.

At last, we illustrate the reduced chemical potential $\mu$ for both the symmetric and asymmetric cases in Fig. 6 It can be seen that the chemical potential monotonously increases with the interaction strength for all cases. For the symmetric-potential and equal-population case, the two components have the same chemical potential, even if they are spatially separated in the ferromagnetic state. In contrast, for the asymmetric-potential and equal-population case, chemical potentials of the two components have the same value at first, and then become different when the two components start to separate (see the black arrow in Fig. 6). In the case of unequal-population, each component has different chemical potential. Comparing Fig. 4 with Fig. [5] it seems that the density profiles for the two asymmetric cases display some similar features when the interaction is strong enough, as shown in Figs. $4 \mathrm{~d}$ and $5 \mathrm{f}$. 


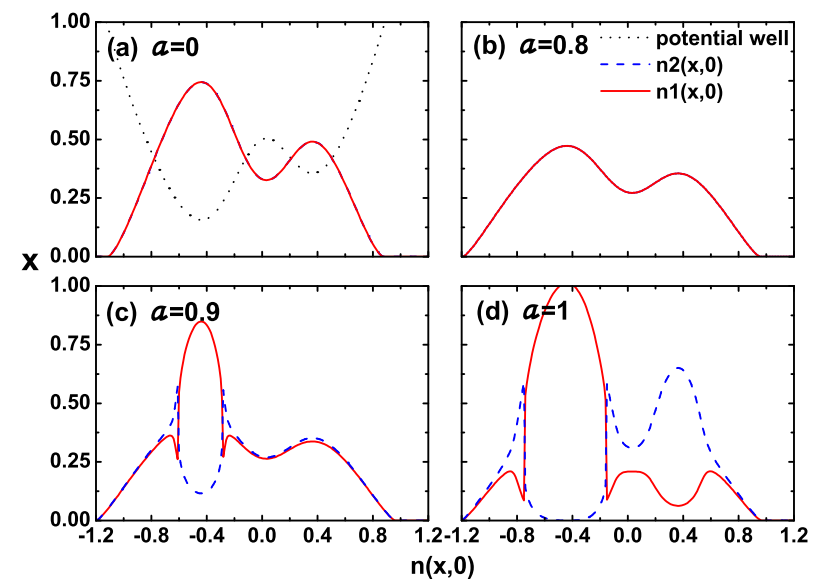

Fig. 5: Density profile along the axial coordinate $x$ with radial coordinate $\rho=0$ in the asymmetric double-well potential $U(x, \rho)=x^{2}+0.5 e^{-10 x^{2}}+0.5 \sin (0.5 x)+\rho^{2}$. The reduced interaction strength $a$ from $(a)$ to $(d)$ is $0,0.8,0.9,1$. The dotted-black curve illustrates the potential well. The solid-red and dashed-blue curves represent different components.

Conclusion. - In conclusion, we have studied density profiles of two-component Fermi gases in double wells using density functional theory. Both symmetric and asymmetric DW potentials have been taken into account and the unequal-population Fermi gas in the symmetric DW trap has also been discussed. For all the cases, the obtained results indicate that the two components can be spatially separated if the repulsive interaction becomes strong enough. This implies that the ferromagnet aligns in the $\mathrm{z}$ (population-imbalance) direction. In the ferromagnetic state, each component tends to gather in one well and thus its population dominates in this well. Therefore, the occurrence of the FM transition can be examined by measuring atomic populations in one well. It is much easier than detecting magnetic domains in situ.

Finally, we note that our calculations may not be directly applicable when the ferromagnetic alignment is in an in-plane direction. Moreover, the present study can be extended in several directions. The critical point needs to be calculated more accurately. Calculations beyond mean-field theory and numerical simulations reveal a lower $k_{F} a \approx 0.8-1.1$ in the single well 12 . It is natural to expect that it is also the case in the double well. Recent works also suggested that some nontrivial effects may play important role in understanding the FM transition, such as spatially modulated magnetism [13, pairing effect [14, and spin fluctuations [15].

$$
* * *
$$

This work was supported by the National Natural Science Foundation of China (Grant No. 11074021), the Key Project of the Chinese Ministry of Education (No. 109011), and the Fundamental Research Funds for

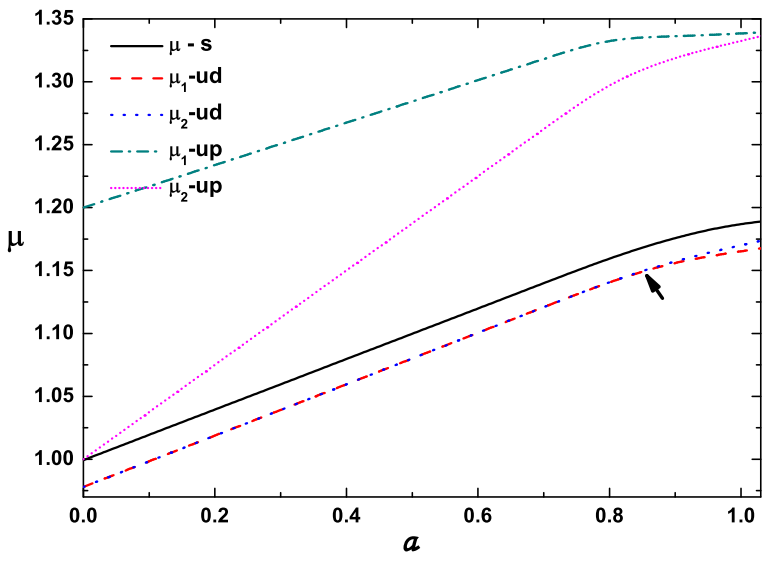

Fig. 6: The reduced chemical potential $\mu$ versus the interaction parameter $a$ for different cases. Here $s$ is short for symmetric case, while $u d$ and up correspond to unequal depth of potential well and unequal population. Note that $\mu=\mu_{1}=\mu_{2}$ for $s$ case and the black arrow shows the transition point in the $u d$ case.

the Central Universities of China. The authors thank Yajiang Hao and Joachim Brand for helpful discussions.

\section{REFERENCES}

[1] Mohn P. (Editor), Magnetism in the Solid state: An Introduction (Springer-Verlag, Berlin) 2003.

[2] Stamper-Kurn D. M., Andrews M. R., Chikkatur A. P., Inouye S., Miesner H.-J., Stenger J. and Ketterle W., Phys. Rev. Lett., 80 (1998) 2027; Stenger J., Inouye S., Stamper-Kurn D. M., Miesner H.-J., Chikkatur A. P. and Ketterle W., Nature, 396 (1998) 345 .

[3] Sadler L. E., Higbie J. M., Leslie S. R., Vengalattore M. and Stamper-Kurn D. M., Nature, 443 (2006) 312.

[4] Ho T. L., Phys. Rev. Lett., 81 (1998) 742.

[5] Ohmi T. and Machida K., J. Phys. Soc. Jpn., 67 (1998) 1822.

[6] Gu Q. and Kremm R. A., Phys. Rev. A, 68 (2003) 031604(R); Gu Q., Bongs K. and Sengstock K., Phys. Rev. A, 70 (2004) 063609; TaO C., Wang P., Qin J. and Gu Q., Phys. Rev. B, 78 (2008) 134403.

[7] Kis-Szabo K., Szepfalusy P. and Szirmai G., Phys. Rev. A, 72 (2005) 023617; Szirmai G., Kis-Szabo K., and Szepfalusy P., Eur. Phys. J. D, 36 (2005) 281.

[8] Chin C., Grimm R., Julienne P. and Tiesinga E., Rev. Mod. Phys., 82 (2010) 1225.

[9] Jo G.-B., Lee Y.-R., Choi J.-H., Christensen C. A., Kim T. H., Thywissen J. H., Pritchard D. E. and Ketterle W., Science, 325 (2009) 1521.

[10] Duine R. A. and MacDonald A. H., Phys. Rev. Lett., 95 (2005) 230403.

[11] LeBlanc L. J., Thywissen J. H., Burkov A. A. and Paramekanti A., Phys. Rev. A, 80 (2009) 013607.

[12] Conduit G. J. and Simons B. D., Phy. Rev. Lett., 103 (2009) 200403; Pilati S., Bertaina G., Giorgini S. and Troyer M., Phy. Rev. Lett., 105 (2010) 030405; Chang 
S. Y., Randeria M. and Trivedi N., Proceedings of the National Academy of Sciences, 108 (2011) 51.

[13] Conduit G. J., Green A. G. and Simons B. D., Phy. Rev. Lett., 103 (2009) 207201.

[14] Pekker D., Babadi M., Sensarma R., Zinner N., Pollet L., Zwierlein M. W. and Demler E., Phy. Rev. Lett., 106 (2011) 050402.

[15] Recati A. and Stringari S., Phys. Rev. Lett., 106 (2011) 080402.

[16] Conduit G. J. and Simons B. D., Phy. Rev. A, 79 (2009) 053606 .

[17] Zhai H., Phys. Rev. A, 80 (2009) 051605(R).

[18] Amoruso M., Meccoli I., Minguzzi A. and Tosi M. P., Eur. Phys. J. D, 8 (2000) 361.

[19] Salasnich L., Pozzi B., Parola A. and Reatto L., J. Phys. B, 33 (2000) 3943.

[20] Sogo T. and Yabu H., Phys. Rev. A, 66 (2002) 043611.

[21] Jo G.-B., Lee Y.-R., Choi J.-H., Christensen C. A., Kim T. H., Thywissen J. H., Pritchard D. E. and KetTerle W., e-print arXiv: 0910.3419, (2009).

[22] Berdnikov I., Coleman P. and Simon S. H., Phys. Rev. $B, 79$ (2009) 224403.

[23] Duine R. A., Polini M., Stoof H. T. C. and Vignale G., Phys. Rev. Lett., 104 (2010) 220403.

[24] Butts D. A. and Rokhsar D. S., Phys. Rev. A, 55 (1997) 4346. 